\title{
The generalized 3-connectivity of lexicographic product graphs**
}

\author{
Xueliang Li $\|^{\Uparrow}$ and Yaping Mao ${ }^{1,2}$ 非 \\ ${ }^{1}$ Center for Combinatorics and LPMC-TJKLC, Nankai University, Tianjin 300071, China \\ ${ }^{2}$ Department of Mathematics, Qinghai Normal University, Xining, Qianghai 810008, China
}

received 12 $2^{\text {th }}$ Aug. 2013, revised 26 th Dec. 2013, 10 th Apr. 2014, accepted $17^{\text {th }}$ May 2014.

The generalized $k$-connectivity $\kappa_{k}(G)$ of a graph $G$, first introduced by Hager, is a natural generalization of the concept of (vertex-)connectivity. Denote by $G \circ H$ and $G \square H$ the lexicographic product and Cartesian product of two graphs $G$ and $H$, respectively. In this paper, we prove that for any two connected graphs $G$ and $H, \kappa_{3}(G \circ H) \geq$ $\kappa_{3}(G)|V(H)|$. We also give upper bounds for $\kappa_{3}(G \square H)$ and $\kappa_{3}(G \circ H)$. Moreover, all the bounds are sharp.

Keywords: Connectivity, Steiner tree, Internally disjoint Steiner trees, Packing, Generalized connectivity, Lexicographic product.

\section{Introduction}

All graphs considered in this paper are undirected, finite and simple. We refer to the book [4] for graph theoretical notation and terminology not described here. For a graph $G$, let $V(G), E(G)$ and $\delta(G)$ denote the set of vertices, the set of edges and the minimum degree of $G$, respectively. As usual, $|V(G)|$ is called the order of $G$. For $S \subseteq V(G)$, we denote by $G \backslash S$ the subgraph obtained by deleting from $G$ the vertices of $S$ together with the edges incident with them. We divide our introduction into the following four subsections to state the motivations and our results of this paper.

\subsection{Connectivity and its generalizations}

Connectivity is one of the most basic concepts in graph theory, both in combinatorial sense and in algorithmic sense. The classical connectivity has two equivalent definitions. The connectivity of $G$, written $\kappa(G)$, is the minimum size of a vertex set $S \subseteq V(G)$ such that $G \backslash S$ is disconnected or has only one vertex. We call this definition the 'cut' version definition of connectivity. A well-known theorem of Whitney [43]

\footnotetext{
${ }^{*}$ Supported by NSFC No. 11371205 and PCSIRT.

${ }^{\dagger}$ Email: lxlenankai.edu.cn

‡Email: maoyaping@ymail.com

1365-8050 @ 2014 Discrete Mathematics and Theoretical Computer Science (DMTCS), Nancy, France
} 
provides an equivalent definition of connectivity, which can be called the 'path' version definition of connectivity. For any two distinct vertices $x$ and $y$ in $G$, the local connectivity $\kappa_{G}(x, y)$ is the maximum number of internally disjoint paths connecting $x$ and $y$. Then $\kappa(G)=\min \left\{\kappa_{G}(x, y) \mid x, y \in V(G), x \neq y\right\}$ is defined to be the connectivity of $G$.

Although there are many elegant and powerful results on connectivity in graph theory, the basic notation of classical connectivity may not be general enough to capture some computational settings. So people tried to generalize this concept. For the 'cut' version definition of connectivity, we find that the above minimum vertex set does not regard to the number of components of $G \backslash S$. Two graphs with the same connectivity may have different degrees of vulnerability in the sense that the deletion of a vertex cut-set of minimum cardinality from one graph may produce a graph with considerably more components than in the case of the other graph. For example, the star $K_{1, n}$ and the path $P_{n+1}(n \geq 3)$ are both trees of order $n+1$ and therefore connectivity 1 , but the deletion of a cut-vertex from $K_{1, n}$ produces a graph with $n$ components while the deletion of a cut-vertex from $P_{n+1}$ produces only two components. Chartrand et al. [5] generalized the 'cut' version definition of connectivity. For an integer $k(k \geq 2)$ and a graph $G$ of order $n(n \geq k)$, the $k$-connectivity $\kappa_{k}^{\prime}(G)$ is the smallest number of vertices whose removal from $G$ of order $n(n \geq k)$ produces a graph with at least $k$ components or a graph with fewer than $k$ vertices. Thus, for $k=2, \kappa_{2}^{\prime}(G)=\kappa(G)$. For more details about $k$-connectivity, we refer to [5, 8, 34, 35].

The generalized connectivity of a graph $G$, mentioned by Hager in [16], is a natural generalization of the 'path' version definition of connectivity. For a graph $G=(V, E)$ and a set $S \subseteq V(G)$ of at least two vertices, an $S$-Steiner tree or a Steiner tree connecting $S$ (or simply, an $S$-tree) is a such subgraph $T=\left(V^{\prime}, E^{\prime}\right)$ of $G$ that is a tree with $S \subseteq V^{\prime}$. Note that when $|S|=2$ a minimal Steiner tree connecting $S$ is just a path connecting the two vertices of $S$. Two Steiner trees $T$ and $T^{\prime}$ connecting $S$ are said to be internally disjoint if $E(T) \cap E\left(T^{\prime}\right)=\emptyset$ and $V(T) \cap V\left(T^{\prime}\right)=S$. For $S \subseteq V(G)$ and $|S| \geq 2$, the generalized local connectivity $\kappa_{G}(S)$ is the maximum number of internally disjoint trees connecting $S$ in $G$, that is, we search for the maximum cardinality of edge-disjoint trees which contain $S$ and are vertex-disjoint with the exception of the vertices in $S$. For an integer $k$ with $2 \leq k \leq n$, the generalized $k$-connectivity (or $k$-tree-connectivity) is defined as $\kappa_{k}(G)=\min \left\{\kappa_{G}(S)|S \subseteq V(G)| S \mid,=k\right\}$, that is, $\kappa_{k}(G)$ is the minimum value of $\kappa_{G}(S)$ when $S$ runs over all $k$-subsets of $V(G)$. Clearly, when $|S|=2$, $\kappa_{2}(G)$ is nothing new but the connectivity $\kappa(G)$ of $G$, that is, $\kappa_{2}(G)=\kappa(G)$, which is the reason why one addresses $\kappa_{k}(G)$ as the generalized connectivity of $G$. By convention, for a connected graph $G$ with less than $k$ vertices, we set $\kappa_{k}(G)=1$, and $\kappa_{k}(G)=0$ when $G$ is disconnected.

Note that the generalized $k$-connectivity and the $k$-connectivity of a graph are indeed different. Take for example, the graph $G_{0}$ obtained from a triangle with vertex set $\left\{v_{1}, v_{2}, v_{3}\right\}$ by adding three new vertices $u_{1}, u_{2}, u_{3}$ and joining $v_{i}$ to $u_{i}$ by an edge for $1 \leq i \leq 3$. Then $\kappa_{3}\left(G_{0}\right)=1$ but $\kappa_{3}^{\prime}\left(G_{0}\right)=2$. There are many results on the generalized $k$-connectivity; see $[6,15,23,24,25,26,27,28,29,30,31,36]$.

\subsection{The application background of generalized connectivity}

One extreme of $\kappa_{k}(G)$ is when $k=2$. As we mentioned in the last subsection, $\kappa_{2}(G)=\kappa(G)$ is just the connectivity of a graph $G$. Another extreme of $\kappa_{k}(G)$ is when $k=n$. For $k=n$, one can see that $S=V(G)$ and $\kappa_{n}(G)$ is just the maximum number of edge-disjoint spanning trees in $G$ (For $k=n$, each Steiner tree connecting $S$ is a spanning tree of $G$ ). Then $\kappa_{n}(G)$ is called the spanning-tree packing number of $G$. For the spanning-tree packing number, we refer to [37, 38]. For a given graph $G$, the problem of finding out the spanning-tree packing number of $G$ is called the spanning tree packing problem. Note that spanning tree packing problem is a special case of the generalized $k$-connectivity. 
Besides being of theoretical interest, spanning tree packing problem has its practical applications. One of them is to enhance the ability of fault tolerance in a network; see [12, 20]. Consider a source node $u$ that wants to broadcast a message on a network with $\ell$ edge-disjoint spanning trees. The node $u$ copies $\ell$ messages to different spanning trees. If there are no more than $\ell-1$ fault edges, all the other nodes can receive the message. In fact, the generalized $k$-connectivity $\kappa_{k}(G)$, which is a generalization of the spanning-tree packing number $\kappa_{n}(G)$, can be seen as another parameter to express the ability of fault tolerance of a network, where any two Steiner trees connecting the node set $S$ in $G$ are required to share no Steiner vertex (Each vertex in $V(G) \backslash S$ is called a Steiner vertex).

In addition to being a natural combinatorial measure, the generalized $k$-connectivity can be motivated by its interesting interpretation in practice. For example, suppose that $G$ represents a network. If one considers to connect a pair of vertices of $G$, then a path is used to connect them. However, if one wants to connect a set $S$ of vertices of $G$ with $|S| \geq 3$, then a tree has to be used to connect them. This kind of tree for connecting a set of vertices is usually called a Steiner tree, and popularly used in the physical design of VLSI circuits (see [13, 14, 40]). In this application, a Steiner tree is needed to share an electric signal by a set of terminal nodes. Steiner tree is also used in computer communication networks (see [10]) and optical wireless communication networks (see [7]). Usually, one wants to consider how tough a network can be, for the connection of a set of vertices. Then, the number of totally independent ways to connect them is a measure for this purpose. The generalized $k$-connectivity can serve for measuring the capability of a network $G$ to connect any $k$ vertices in $G$.

\subsection{Graph products and the parameters $\kappa$ and $\kappa_{n}$}

The Cartesian product of two graphs $G$ and $H$, written as $G \square H$, is the graph with vertex set $V(G) \times$ $V(H)$, in which two vertices $(u, v)$ and $\left(u^{\prime}, v^{\prime}\right)$ are adjacent if and only if $u=u^{\prime}$ and $\left(v, v^{\prime}\right) \in E(H)$, or $v=v^{\prime}$ and $\left(u, u^{\prime}\right) \in E(G)$. Clearly, the Cartesian product is commutative, that is, $G \square H$ is isomorphic to $H \square G$. The lexicographic product of two graphs $G$ and $H$, written as $G \circ H$, is defined as follows: $V(G \circ H)=V(G) \times V(H)$, and two distinct vertices $(u, v)$ and $\left(u^{\prime}, v^{\prime}\right)$ of $G \circ H$ are adjacent if and only if either $\left(u, u^{\prime}\right) \in E(G)$ or $u=u^{\prime}$ and $\left(v, v^{\prime}\right) \in E(H)$. Note that unlike the Cartesian product, the lexicographic product is a non-commutative product since $G \circ H$ is usually not isomorphic to $H \circ G$.

Product networks were proposed based upon the idea of using the cross product as a tool for "combining" two known graphs with established properties to obtain a new one that inherits properties from both [9]. There has been an increasing interest in a class of interconnection networks called Cartesian product networks; see [1, 9, 21]. In [21], Ku et al. studied the problem of constructing the maximum number of edge-disjoint spanning trees in Cartesian product networks, and gave a sharp lower bound of $\kappa_{n}(G \square H)$. A natural question is to study sharp upper and lower bounds of $\kappa_{k}(G \square H)$. For $k=2$, Sabidussi [39] derived the following theorem on the connectivity of Cartesian product graphs.

Theorem 1.1 [39] Let $G$ and $H$ be two connected graphs. Then $\kappa(G \square H) \geq \kappa(G)+\kappa(H)$.

Lexicographic product is also studied extensively; see [18]. Recently, some applications in networks of the lexicographic product were studied; see [3, 11, 22]. Here we will study the sharp upper and lower bounds of $\kappa_{k}(G \circ H)$. For $k=2$, Yang and $\mathrm{Xu}$ [44] investigated the classical connectivity of the lexicographic product of two graphs.

Theorem 1.2 [44] Let $G$ and $H$ be two graphs. If $G$ is non-trivial, non-complete and connected, then $\kappa(G \circ H)=\kappa(G)|V(H)|$. 


\subsection{Graph products and the parameter $\kappa_{3}$}

In [25], Li et al. studied the generalized 3-connectivity of Cartesian product graphs and got a lower bound of it. Their result could be seen as an extension of Sabidussi's theorem.

Theorem 1.3 [25] Let $G$ and $H$ be connected graphs such that $\kappa_{3}(G) \geq \kappa_{3}(H)$. The following assertions hold:

(i) If $\kappa(G)=\kappa_{3}(G)$, then $\kappa_{3}(G \square H) \geq \kappa_{3}(G)+\kappa_{3}(H)-1$. Moreover, the bound is sharp;

(ii) If $\kappa(G)>\kappa_{3}(G)$, then $\kappa_{3}(G \square H) \geq \kappa_{3}(G)+\kappa_{3}(H)$. Moreover, the bound is sharp.

In Section 2, we obtain the following lower bound of $\kappa_{3}(G \circ H)$, which is the main result of this paper. This result could be seen as an extension of Theorem 1.2 .

Theorem 1.4 Let $G$ and $H$ be two connected graphs. Then

$$
\kappa_{3}(G \circ H) \geq \kappa_{3}(G)|V(H)| .
$$

Moreover, the bound is sharp.

In Section 3, we derive the upper bounds of $\kappa_{3}(G \circ H)$ and $\kappa_{3}(G \square H)$.

Theorem 1.5 Let $G$ and $H$ be two connected graphs. If $G$ is non-trivial and non-complete, then $\kappa_{3}(G \circ$ $H) \leq\left\lfloor\frac{4}{3} \kappa_{3}(G)+r-\frac{4}{3}\left\lceil\frac{r}{2}\right\rceil\right\rfloor|V(H)|$, where $r \equiv \kappa(G)(\bmod 4)$. Moreover, the bound is sharp.

Theorem 1.6 Let $G$ and $H$ be two connected graphs. Then $\kappa_{3}(G \square H) \leq \min \left\{\left\lfloor\frac{4}{3} \kappa_{3}(G)+r_{1}-\frac{4}{3}\left\lceil\frac{r_{1}}{2}\right\rceil\right\rfloor\right.$ $\left.|V(H)|,\left\lfloor\frac{4}{3} \kappa_{3}(H)+r_{2}-\frac{4}{3}\left\lceil\frac{r_{2}}{2}\right\rceil\right\rfloor|V(G)|, \delta(G)+\delta(H)\right\}$, where $r_{1} \equiv \kappa(G)(\bmod 4)$ and $r_{2} \equiv \kappa(H)(\bmod 4)$. Moreover, the bound is sharp.

\section{Lower bound of $\kappa_{3}(G \circ H)$}

In this section, let $G$ and $H$ be two connected graphs with $V(G)=\left\{u_{1}, u_{2}, \ldots, u_{n}\right\}$ and $V(H)=$ $\left\{v_{1}, v_{2}, \ldots, v_{m}\right\}$, respectively. Then $V(G \circ H)=\left\{\left(u_{i}, v_{j}\right) \mid 1 \leq i \leq n, 1 \leq j \leq m\right\}$. For $v \in V(H)$, we use $G(v)$ to denote the subgraph of $G \circ H$ induced by the vertex set $\left\{\left(u_{i}, v\right) \mid 1 \leq i \leq n\right\}$. Similarly, for $u \in V(G)$, we use $H(u)$ to denote the subgraph of $G \circ H$ induced by the vertex set $\left\{\left(u, v_{j}\right) \mid 1 \leq j \leq m\right\}$. In the sequel, let $K_{s, t}, K_{n}$ and $P_{n}$ denote the complete bipartite graph of order $s+t$ with part sizes $s$ and $t$, complete graph of order $n$, and path of order $n$, respectively. If $G$ is a connected graph and $x, y \in V(G)$, then the distance $d_{G}(x, y)$ between $x$ and $y$ is the length of a shortest path connecting $x$ and $y$ in $G$. The degree of a vertex $v$ in $G$ is denoted by $d_{G}(v)$.

We now introduce the general idea of the proof of Theorem 1.4, with a running example (corresponding to Fig. 1). From the definition, the lexicographic product graph $G \circ H$ is a graph obtained by replacing each vertex of $G$ by a copy of $H$ and replacing each edge of $G$ by a complete bipartite graph $K_{m, m}$. Recall that $V(G)=\left\{u_{1}, u_{2}, \ldots, u_{n}\right\}$. Clearly, $V(G \circ H)=\bigcup_{i=1}^{n} V\left(H\left(u_{i}\right)\right)$. Take for example, let $G=K_{4}$ (see Fig. $1(a)$ ). Set $V\left(K_{4}\right)=\left\{u_{1}, u_{2}, u_{3}, u_{4}\right\}$ and $|V(H)|=m$. Then $K_{4} \circ H$ is a graph obtained by replacing each vertex of $K_{4}$ by a copy of $H$ and replacing each edge of $K_{4}$ by a complete bipartite graph $K_{m, m}$ (see Fig. $1(e)$ ). Clearly, $V\left(K_{4} \circ H\right)=\bigcup_{i=1}^{4} V\left(H\left(u_{i}\right)\right)$ (see Fig. $1(e)$ ).

In this section, we give the proof of Theorem 1.4 For two connected graphs $G$ and $H$, we prove that $\kappa_{3}(G \circ H) \geq \kappa_{3}(G)|V(H)|$. Set $\kappa_{3}(G)=\ell$ and $|V(H)|=m$. From the definition of $\kappa_{3}(G \circ H)$, it suffices to show that $\kappa_{G \circ H}(S) \geq \ell m$ for any $S \subseteq V(G \circ H)$ and $|S|=3$. From the definition of 
$\kappa_{G \circ H}(S)$, we need to find out $\ell m$ internally disjoint Steiner trees connecting $S$ in $G \circ H$. Let $S=$ $\{x, y, z\}$. Recall that $V(G)=\left\{u_{1}, u_{2}, \ldots, u_{n}\right\}$. From the above analysis, we know that $x, y, z \in$ $V(G \circ H)=\bigcup_{i=1}^{n} V\left(H\left(u_{i}\right)\right)$. Without loss of generality, let $x \in H\left(u_{i}\right), y \in H\left(u_{j}\right)$ and $z \in H\left(u_{k}\right)$ (note that $u_{i}, u_{j}, u_{k}$ are not necessarily different). For the above example, we have $x, y, z \in V\left(K_{4} \circ H\right)=$ $\bigcup_{i=1}^{4} V\left(H\left(u_{i}\right)\right)$. Without loss of generality, let $x \in H\left(u_{1}\right), y \in H\left(u_{2}\right)$ and $z \in H\left(u_{3}\right)$ (see Fig. $1(e)$ ).

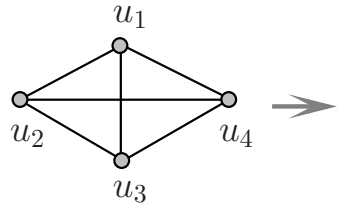

(a) $G$

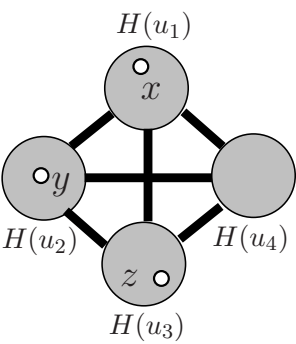

(e) $G \circ H$

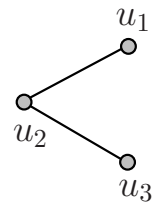

(b) $T_{1}$

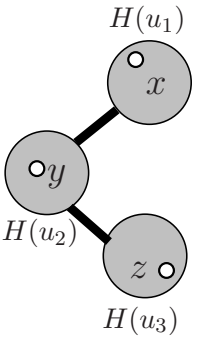

(f) $T_{1} \circ H$

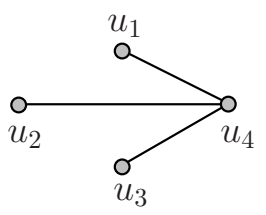

(c) $T_{2}$

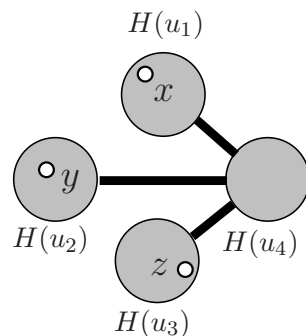

(g) $T_{2} \circ H$

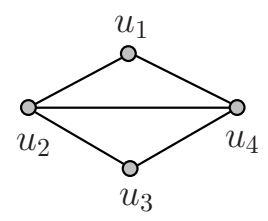

(d) $T_{1} \cup T_{2}$

Fig. 1: An example for $G \circ H$.

Because $u_{i}, u_{j}, u_{k} \in V(G)$ and $\kappa_{3}(G)=\ell$, there are $\ell$ internally disjoint Steiner trees connecting $\left\{u_{i}, u_{j}, u_{k}\right\}$, say $T_{1}, T_{2}, \cdots, T_{\ell}$. Note that $\bigcup_{i=1}^{\ell} T_{i}$ is a subgraph of $G$. Thus $\left(\bigcup_{i=1}^{\ell} T_{i}\right) \circ H$ is a subgraph of $G \circ H$. For the above example, we have $\kappa_{3}(G)=\kappa_{3}\left(K_{4}\right)=\ell=2$. It suffices to prove that $\kappa_{3}(G \circ H) \geq \kappa_{3}\left(K_{4}\right)|V(H)|=2 m$. Then there are $\ell=2$ internally disjoint Steiner trees connecting $\left\{u_{1}, u_{2}, u_{3}\right\}$, say $T_{1}, T_{2}$ (see Fig. $1(b),(c)$ ). Note that $T_{1} \cup T_{2}$ is a subgraph of $G$ (see Fig. $1(a),(d)$ ). Then $\left(T_{1} \cup T_{2}\right) \circ H$ is a subgraph of $G \circ H$ (see Fig. $1(e),(h)$ ).

If we can prove that $\kappa_{\left(\bigcup_{i=1}^{\ell} T_{i}\right) \circ H}(S) \geq \ell m$ for $S=\{x, y, z\}$, then $\kappa_{G \circ H}(S) \geq \kappa_{\left(\bigcup_{i=1}^{\ell} T_{i}\right) \circ H}(S) \geq$ $\ell m$ since $\left(\bigcup_{i=1}^{\ell} T_{i}\right) \circ H$ is a subgraph of $G \circ H$. Therefore, the problem is converted into finding out $\ell m$ internally disjoint Steiner trees connecting $S$ in $\left(\bigcup_{i=1}^{\ell} T_{i}\right) \circ H$. Observe that $\left(\bigcup_{i=1}^{\ell} T_{i}\right) \circ H=$ $\bigcup_{i=1}^{\ell}\left(T_{i} \circ H\right)$. The structure of $\bigcup_{i=1}^{\ell}\left(T_{i} \circ H\right)$ is shown in Fig. 2. In order to show this structure clearly, we take $\ell$ copies of $H\left(u_{j}\right)$, and $\ell$ copies of $H\left(u_{k}\right)$. Note that, these $\ell$ copes of $H\left(u_{j}\right)$ (resp. $H\left(u_{k}\right)$ ) represent the same graph. For the above example, if we can prove that $\kappa_{\left(T_{1} \cup T_{2}\right) \circ H}(S) \geq 2 m$ for $S=\{x, y, z\}$, then $\kappa_{G \circ H}(S) \geq \kappa_{\left(T_{1} \cup T_{2}\right) \circ H}(S) \geq 2 m$, as desired. Note that $\left(T_{1} \cup T_{2}\right) \circ H=\left(T_{1} \circ H\right) \cup\left(T_{2} \circ H\right)$. The problem is converted into finding out $2 m$ internally disjoint Steiner trees connecting $S$ in $\left(T_{1} \circ H\right) \cup\left(T_{2} \circ H\right)$ (see Fig. $1(h)$ ). 
For each $T_{i}(1 \leq i \leq \ell)$, if we can find out $m$ internally disjoint Steiner trees connecting $S$ in $T_{i} \circ H$, say $T_{i, 1}, T_{i, 2}, \cdots, T_{i, m}$, then the total number of internally disjoint Steiner trees connecting $S$ in $\bigcup_{i=1}^{k}\left(T_{i} \circ\right.$ $H)=\left(\bigcup_{i=1}^{\ell} T_{i}\right) \circ H$ are $\ell m$, which implies that $\kappa_{G \circ H}(S) \geq \kappa_{\left(\bigcup_{i=1}^{\ell} T_{i}\right) \circ H}(S) \geq \ell m$ (Note that we must guarantee that any two trees of $\left\{T_{i, j} \mid 1 \leq i \leq \ell, 1 \leq j \leq m\right\}$ are internally disjoint). Furthermore, from the arbitrariness of $S$, we can get $\kappa_{3}(G \circ H) \geq \ell m=\kappa_{3}(G)|V(H)|$ and complete the proof of Theorem 1.4. For the above example, we need to find out $m$ internally disjoint Steiner trees connecting $S$ in both $T_{1} \circ H$ and $T_{2} \circ H$ (see Fig. $1(f),(g)$ ). Then the total number of internally disjoint Steiner trees connecting $S$ in $\left(T_{1} \cup T_{2}\right) \circ H=\left(T_{1} \circ H\right) \cup\left(T_{2} \circ H\right)$ are $2 m$, which implies that $\kappa_{G \circ H}(S) \geq \kappa_{\left(T_{1} \cup T_{2}\right) \circ H}(S) \geq 2 m$. Thus the result follows by the arbitrariness of $S$.

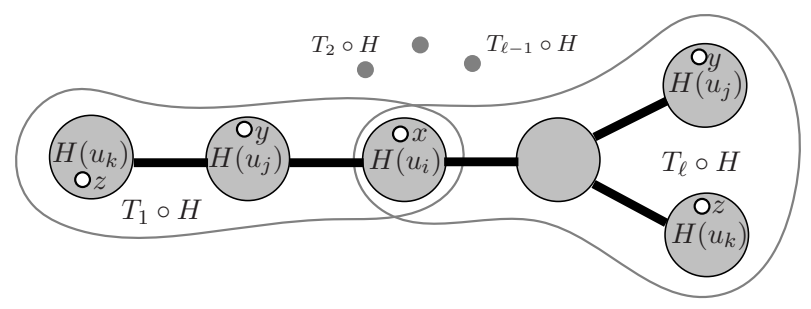

Fig. 2: The structure of $\left(\bigcup_{i=1}^{k} T_{i}\right) \circ H=\bigcup_{i=1}^{k}\left(T_{i} \circ H\right)$.

From the above analysis, we need to consider the graph $T \circ H$ and prove that for any $S=\{x, y, z\} \subseteq$ $V(T \circ H)$ there are $m$ internally disjoint Steiner trees connecting $S$ in $T \circ H$, where $T$ is a Steiner tree connecting $\left\{u_{i}, u_{j}, u_{k}\right\}$ in $G$. If so, then $\kappa_{T \circ H}(S) \geq m$ for any $S=\{x, y, z\} \subseteq V(T \circ H)$, which implies that $\kappa_{3}(T \circ H) \geq m=|V(H)|$.

In the basis of such an idea, we study the generalized 3-connectivity of the lexicographic product of a tree $T$ and a connected graph $H$ first, and show that $\kappa_{3}(T \circ H) \geq|V(H)|$ in Subsection 2.2. After this preparation, we consider the graph $G \circ H$ where $G$ is a general (connected) graph and prove $\kappa_{3}(G \circ H) \geq \kappa_{3}(G)|V(H)|$ in Subsection 2.3. In Subsection 2.1, we investigate the generalized 3connectivity of the lexicographic product of a path $P_{n}$ and a connected graph $H$ since a path is a special tree. So the proof of Theorem 1.4 can be divided into the above mentioned three subsections. Each previous subsection is a preparation of the subsequent one.

Before realizing the above three steps, we introduce the following two well known lemmas, which will be used later.

Given a vertex $x$ and a set $U$ of vertices, an $(x, U)$-fan is a set of paths from $x$ to $U$ such that any two of them share only the vertex $x$. The size of a $(x, U)$-fan is the number of internally disjoint paths from $x$ to $U$.

Lemma 2.1 (Fan Lemma, [42], p-170) A graph is $k$-connected if and only if it has at least $k+1$ vertices and, for every choice of $x, U$ with $|U| \geq k$, it has an $(x, U)$-fan of size $k$.

Lemma 2.2 (Expansion Lemma, [42], p-162) If $G$ is a k-connected graph, and $G^{\prime}$ is obtained from $G$ by adding a new vertex $y$ with at least $k$ neighbors in $G$, then $G^{\prime}$ is a $k$-connected.

Let $G$ be a $k$-connected graph. Choose $U \subseteq V(G)$ with $|U|=k$. Then the graph $G^{\prime}$ is obtained from $G$ by adding a new vertex $y$ and joining each vertex of $U$ and the vertex $y$. We call this operation an 
expansion operation at $y$ and $U$. Denote the resulting graph $G^{\prime}$ by $G^{\prime}=G \vee\{y, U\}$.

\subsection{The Lexicographic product of a path and a connected graph}

To start with, we show the following proposition, which is a preparation of the next subsection.

Proposition 2.3 Let $H$ be a connected graph and $P_{n}$ be a path with $n$ vertices. Then $\kappa_{3}\left(P_{n} \circ H\right) \geq$ $|V(H)|$. Moreover, the bound is sharp.

Set $V(H)=\left\{v_{1}, v_{2}, \ldots, v_{m}\right\}$ and $V\left(P_{n}\right)=\left\{u_{1}, u_{2}, \ldots, u_{n}\right\}$. Let $u_{1}, u_{2}, \cdots, u_{n}$ be the linear order of vertices on the path $P_{n}$. It suffices to show that $\kappa_{P_{n} \circ H}(S) \geq m$ for any $S=\{x, y, z\} \subseteq V\left(P_{n} \circ H\right)$, that is, there exist $m$ internally disjoint Steiner trees connecting $S$ in $P_{n} \circ H$. We proceed our proof by the following three lemmas.

Lemma 2.4 If $x, y, z$ belongs to the same $V\left(H\left(u_{i}\right)\right)(1 \leq i \leq n)$, then there exist $m$ internally disjoint Steiner trees connecting $S$.

Proof: Without loss of generality, we assume $x, y, z \in V\left(H\left(u_{1}\right)\right)$. Then the trees $T_{j}=x\left(u_{2}, v_{j}\right) \cup$ $y\left(u_{2}, v_{j}\right) \cup z\left(u_{2}, v_{j}\right)(1 \leq j \leq m)$ are $m$ internally disjoint Steiner trees connecting $S$, as desired.

Lemma 2.5 If only two vertices of $\{x, y, z\}$ belong to some copy $H\left(u_{i}\right)(1 \leq i \leq n)$, then there exist $m$ internally disjoint Steiner trees connecting $S$.

Proof: We may assume $x, y \in V\left(H\left(u_{1}\right)\right)$ and $z \in V\left(H\left(u_{i}\right)\right)(2 \leq i \leq n-1)$. In the following argument, we can see that this assumption has no influence on the correctness of our proof. Consider the case $i \geq 3$. Let $P^{\prime}=u_{2} u_{3} \cdots u_{n}$. Clearly, $\kappa\left(P^{\prime} \circ H\right) \geq m$. From Lemma 2.1, there is a $z, U$-fan in $P^{\prime} \circ H$, where $U=V\left(H\left(u_{2}\right)\right)=\left\{\left(u_{2}, v_{j}\right) \mid 1 \leq j \leq m\right\}$. Thus there exist $m$ internally disjoint paths $P_{1}, P_{2}, \cdots, P_{m}$ such that $P_{j}(1 \leq j \leq m)$ is a path connecting $z$ and $\left(u_{2}, v_{j}\right)$. Furthermore, the trees $T_{j}=x\left(u_{2}, v_{j}\right) \cup y\left(u_{2}, v_{j}\right) \cup P_{j}(1 \leq j \leq m)$ are $m$ internally disjoint Steiner trees connecting $S$.

Now we assume $i=2$. We may assume $x, y \in V\left(H\left(u_{1}\right)\right)$ and $z \in V\left(H\left(u_{2}\right)\right)$. Let $x^{\prime}, y^{\prime}$ be the vertices corresponding to $x, y$ in $H\left(u_{2}\right), z^{\prime}$ be the vertex corresponding to $z$ in $H\left(u_{1}\right)$. Clearly, $H\left(u_{1}\right)$ is connected and so there is a path $P_{1}$ connecting $x$ and $y$ in $H\left(u_{1}\right)$.

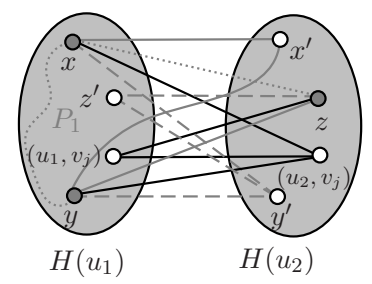

(a)

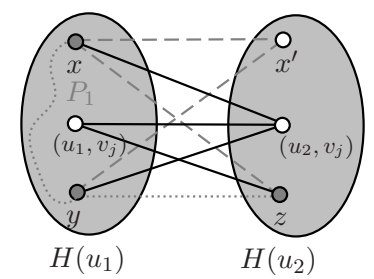

(b)

Fig. 3: Graphs for Lemma 2.5

If $z^{\prime} \notin\{x, y\}$, without loss of generality, let $\left\{x, y, z^{\prime}\right\}=\left\{\left(u_{1}, v_{j}\right) \mid 1 \leq j \leq 3\right\}$ and $\left\{x^{\prime}, y^{\prime}, z\right\}=$ $\left\{\left(u_{2}, v_{j}\right) \mid 1 \leq j \leq 3\right\}$, then the trees $T_{j}=x\left(u_{2}, v_{j}\right) \cup y\left(u_{2}, v_{j}\right) \cup\left(u_{1}, v_{j}\right)\left(u_{2}, v_{j}\right) \cup z\left(u_{1}, v_{j}\right)(4 \leq j \leq m)$ 
and $T_{1}=x x^{\prime} \cup x^{\prime} y \cup y z$ and $T_{2}=x z \cup P_{1}$ and $T_{3}=x y^{\prime} \cup y^{\prime} z^{\prime} \cup y y^{\prime} \cup z z^{\prime}$ are $m$ internally disjoint Steiner trees connecting $S$; see Fig. $3(a)$.

If $z^{\prime} \in\{x, y\}$, without loss of generality, let $z^{\prime}=y,\{x, y\}=\left\{\left(u_{1}, v_{1}\right),\left(u_{1}, v_{2}\right)\right\}$ and $\left\{x^{\prime}, z\right\}=$ $\left\{\left(u_{2}, v_{1}\right),\left(u_{2}, v_{2}\right)\right\}$, then the trees $T_{j}=x\left(u_{2}, v_{j}\right) \cup y\left(u_{2}, v_{j}\right) \cup\left(u_{1}, v_{j}\right)\left(u_{2}, v_{j}\right) \cup z\left(u_{1}, v_{j}\right)(3 \leq j \leq m)$ and $T_{1}=x z \cup x x^{\prime} \cup y x^{\prime}$ and $T_{2}=y z \cup P_{1}$ are $m$ internally disjoint Steiner trees connecting $S$; see Fig. $3(b)$. The proof is complete.

Lemma 2.6 If $x, y, z$ are contained in distinct $H\left(u_{i}\right) s$, then there exist $m$ internally disjoint Steiner trees connecting $S$.

Proof: We have the following cases to consider.

Case 1. $d_{P_{n} \circ H}(x, y)=d_{P_{n} \circ H}(y, z)=1$.

We may assume that $x \in V\left(H\left(u_{1}\right)\right), y \in V\left(H\left(u_{2}\right)\right), z \in V\left(H\left(u_{3}\right)\right)$. In the following argument, we can see that this assumption has no influence on the correctness of our proof. Let $y^{\prime}, z^{\prime}$ be the vertices corresponding to $y, z$ in $H\left(u_{1}\right), x^{\prime}, z^{\prime \prime}$ be the vertices corresponding to $x, z$ in $H\left(u_{2}\right)$ and $x^{\prime \prime}, y^{\prime \prime}$ be the vertices corresponding to $x, y$ in $H\left(u_{3}\right)$.

If $x, y^{\prime}, z^{\prime}$ are distinct vertices in $H\left(u_{1}\right)$, without loss of generality, let $\left\{x, y^{\prime}, z^{\prime}\right\}=\left\{\left(u_{1}, v_{j}\right) \mid 1 \leq\right.$ $j \leq 3\}$ and $\left\{x^{\prime}, y, z^{\prime \prime}\right\}=\left\{\left(u_{2}, v_{j}\right) \mid 1 \leq j \leq 3\right\}$ and $\left\{x^{\prime \prime}, y^{\prime \prime}, z\right\}=\left\{\left(u_{3}, v_{j}\right) \mid 1 \leq j \leq 3\right\}$, then the trees $T_{j}=x\left(u_{2}, v_{j}\right) \cup\left(u_{1}, v_{j}\right)\left(u_{2}, v_{j}\right) \cup y\left(u_{1}, v_{j}\right) \cup z\left(u_{2}, v_{j}\right)(4 \leq j \leq m)$ and $T_{1}=x x^{\prime} \cup x^{\prime} z^{\prime} \cup x^{\prime} z \cup z^{\prime} y$ and $T_{2}=x z^{\prime \prime} \cup z z^{\prime \prime} \cup y^{\prime} z^{\prime \prime} \cup y y^{\prime}$ and $T_{3}=x y \cup y z$ are $m$ internally disjoint Steiner trees connecting $S$; see Fig. $4(a)$.

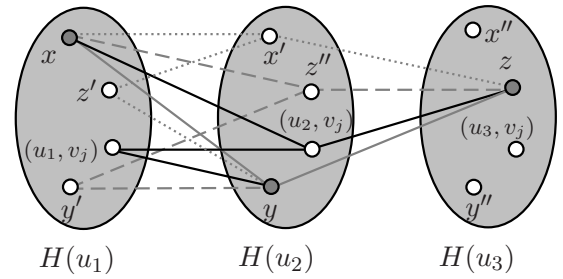

(a)

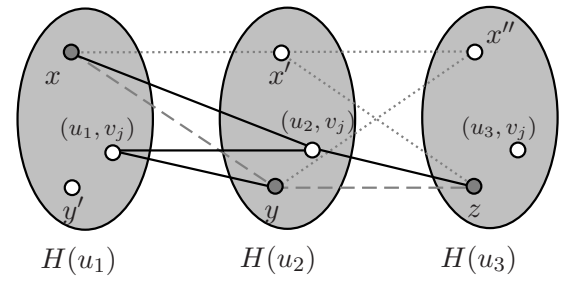

(b)

Fig. 4: Graphs for Case 1 of Lemma 2.6

Suppose that two of $x, y^{\prime}, z^{\prime}$ are the same vertex in $H\left(u_{1}\right)$. If $y^{\prime}=z^{\prime}$, without loss of generality, let $\left\{x, y^{\prime}\right\}=\left\{\left(u_{1}, v_{1}\right),\left(u_{1}, v_{2}\right)\right\}$ and $\left\{x^{\prime}, y\right\}=\left\{\left(u_{2}, v_{1}\right)\right.$,

$\left.\left(u_{2}, v_{2}\right)\right\}$ and $\left\{x^{\prime \prime}, z\right\}=\left\{\left(u_{3}, v_{1}\right),\left(u_{3}, v_{2}\right)\right\}$, then the trees $T_{j}=x\left(u_{2}, v_{j}\right) \cup\left(u_{1}, v_{j}\right)\left(u_{2}, v_{j}\right) \cup y\left(u_{1}, v_{j}\right) \cup$ $z\left(u_{2}, v_{j}\right)(3 \leq j \leq m)$ and $T_{1}=x y \cup y z$ and $T_{2}=x x^{\prime} \cup x^{\prime} x^{\prime \prime} \cup y x^{\prime \prime} \cup x^{\prime} z$ are $m$ internally disjoint Steiner trees connecting $S$; see Fig. $4(b)$. The other cases $\left(x=y^{\prime}\right.$ or $\left.x=z^{\prime}\right)$ can be proved with similar arguments.

Suppose that $x, y^{\prime}, z^{\prime}$ are the same vertex in $H\left(u_{1}\right)$. Without loss of generality, let $x=\left(u_{1}, v_{1}\right)$, $y=\left(u_{2}, v_{1}\right)$ and $z=\left(u_{3}, v_{1}\right)$. Then the trees $T_{j}=x\left(u_{2}, v_{j}\right) \cup\left(u_{1}, v_{j}\right)\left(u_{2}, v_{j}\right) \cup y\left(u_{1}, v_{j}\right) \cup z\left(u_{2}, v_{j}\right)(2 \leq$ $j \leq m)$ and $T_{1}=x y \cup y z$ are $m$ internally disjoint Steiner trees connecting $S$. 
Case 2. $d_{P_{n} \circ H}(x, y)=1$ and $d_{P_{n} \circ H}(y, z) \geq 2$.

We may assume that $x \in V\left(H\left(u_{1}\right)\right), y \in V\left(H\left(u_{2}\right)\right), z \in V\left(H\left(u_{i}\right)\right)(4 \leq i \leq n)$. In the following argument, we can see that this assumption has no influence on the correctness of our proof. Let $y^{\prime}, z^{\prime}$ be the vertices corresponding to $y, z$ in $H\left(u_{1}\right), x^{\prime}, z^{\prime \prime}$ be the vertices corresponding to $x, z$ in $H\left(u_{2}\right)$ and $x^{\prime \prime}, y^{\prime \prime}$ be the vertices corresponding to $x, y$ in $H\left(u_{i}\right)$. Let $P^{\prime}=u_{2} u_{3} \cdots u_{i}$. Clearly, $\kappa\left(P^{\prime} \circ H\right) \geq m$. From Lemma 2.1. there is a $z, U$-fan in $P^{\prime} \circ H$, where $U=V\left(H\left(u_{2}\right)\right)=\left\{\left(u_{2}, v_{j}\right) \mid 1 \leq j \leq m\right\}$. Thus there exist $m$ pairwise internally disjoint paths $P_{1}, P_{2}, \cdots, P_{m}$ such that each $P_{j}(1 \leq j \leq m)$ is a path connecting $z$ and $\left(u_{2}, v_{j}\right)$.

If $x, y^{\prime}, z^{\prime}$ are distinct vertices in $H\left(u_{1}\right)$, without loss of generality, we let $\left\{x, y^{\prime}, z^{\prime}\right\}=\left\{\left(u_{1}, v_{j}\right) \mid 1 \leq\right.$ $j \leq 3\}$ and $\left\{x^{\prime}, y, z^{\prime \prime}\right\}=\left\{\left(u_{2}, v_{j}\right) \mid 1 \leq j \leq 3\right\}$, then the trees $T_{j}=x\left(u_{2}, v_{j}\right) \cup\left(u_{1}, v_{j}\right)\left(u_{2}, v_{j}\right) \cup$ $y\left(u_{1}, v_{j}\right) \cup P_{j}(4 \leq j \leq m)$ and $T_{1}=x x^{\prime} \cup x^{\prime} y^{\prime} \cup y y^{\prime} \cup P_{1}$ and $T_{2}=x y \cup P_{2}$ and $T_{3}=x z^{\prime \prime} \cup z^{\prime} z^{\prime \prime} \cup z^{\prime} y \cup P_{3}$ are $m$ internally disjoint Steiner trees connecting $S$; see Fig. $5(a)$.

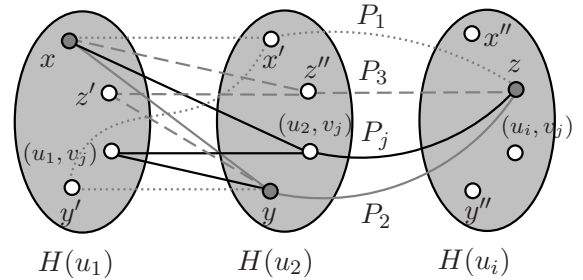

$(a)$

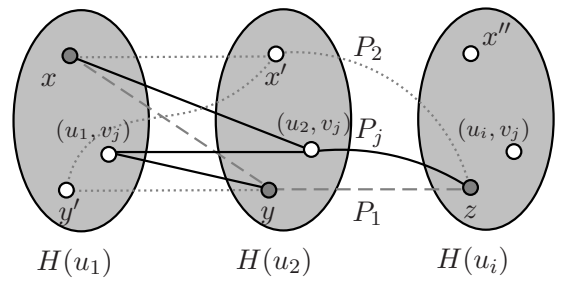

(b)

Fig. 5: Graphs for Case 2 of Lemma 2.6

Suppose that two of $x, y^{\prime}, z^{\prime}$ are the same vertex in $H\left(u_{1}\right)$. If $y^{\prime}=z^{\prime}$, without loss of generality, let $\left\{x, y^{\prime}\right\}=\left\{\left(u_{1}, v_{1}\right),\left(u_{1}, v_{2}\right)\right\}$ and $\left\{x^{\prime}, y\right\}=\left\{\left(u_{2}, v_{1}\right)\right.$,

$\left.\left(u_{2}, v_{2}\right)\right\}$, then the trees $T_{j}=x\left(u_{2}, v_{j}\right) \cup\left(u_{1}, v_{j}\right)\left(u_{2}, v_{j}\right) \cup y\left(u_{1}, v_{j}\right) \cup P_{j}(3 \leq j \leq m)$ and $T_{1}=x y \cup P_{1}$ and $T_{2}=x x^{\prime} \cup x^{\prime} y^{\prime} \cup y y^{\prime} \cup P_{2}$ are $m$ internally disjoint Steiner trees connecting $S$; see Fig. $5(b)$. The other cases $\left(x=y^{\prime}\right.$ or $\left.x=z^{\prime}\right)$ can be proved with similar arguments.

Suppose that $x, y^{\prime}, z^{\prime}$ are the same vertex in $H\left(u_{1}\right)$. Without loss of generality, let $x=\left(u_{1}, v_{1}\right), y=$ $\left(u_{2}, v_{1}\right)$ and $z=\left(u_{i}, v_{1}\right)$. Then the trees $T_{j}=x\left(u_{2}, v_{j}\right) \cup\left(u_{1}, v_{j}\right)\left(u_{2}, v_{j}\right) \cup y\left(u_{1}, v_{j}\right) \cup P_{j}(2 \leq j \leq m)$ and $T_{1}=x y \cup P_{1}$ are $m$ internally disjoint Steiner trees connecting $S$.

Case 3. $d_{P_{n} \circ H}(x, y) \geq 2$ and $d_{P_{n} \circ H}(y, z) \geq 2$.

We may assume that $x \in V\left(H\left(u_{i}\right)\right), y \in V\left(H\left(u_{j}\right)\right), z \in V\left(H\left(u_{k}\right)\right)$, where $i<j<k,|j-i| \geq 2$, $|k-j| \geq 2,1 \leq i \leq n-5,3 \leq j \leq n-2$ and $5 \leq k \leq n$. Let $P^{\prime}=u_{i}, u_{i+1}, \cdots, u_{j-1}$ and $P^{\prime \prime}=u_{j+1}, u_{j+2}, \cdots, u_{k}$. Then $P^{\prime}$ and $P^{\prime \prime}$ are two paths of order at least 2 . Since $\kappa\left(P^{\prime} \circ H\right) \geq m$, from Lemma 2.2 if we add the vertex $y$ to $P^{\prime} \circ H$ and join an edge from $y$ to each $\left(u_{j-1}, v_{r}\right)(1 \leq r \leq m)$, then $\kappa\left(\left(P^{\prime} \circ H\right) \vee\left\{y, V\left(H\left(u_{j-1}\right)\right)\right\}\right) \geq m$. By the same reason, $\kappa\left(\left(P^{\prime \prime} \circ H\right) \vee\left\{y, V\left(H\left(u_{j+1}\right)\right)\right\}\right) \geq m$. From Menger' $\mathrm{s}$ Theorem, there exist $m$ internally disjoint paths connecting $x$ and $y$ in $\left(P^{\prime} \circ H\right) \vee$ $\left\{y, V\left(H\left(u_{j-1}\right)\right)\right\}$, say $P_{1}^{\prime}, P_{2}^{\prime}, \cdots, P_{m}^{\prime}$. Also there exist $m$ internally disjoint paths connecting $y$ and $z$ in $\left(P^{\prime} \circ H\right) \vee\left\{y, V\left(H\left(u_{j+1}\right)\right)\right\}$, say $P_{1}^{\prime \prime}, P_{2}^{\prime \prime}, \cdots, P_{m}^{\prime \prime}$. Note that the union of any path in $\left\{P_{i}^{\prime} \mid 1 \leq i \leq m\right\}$ with any path in $\left\{P_{j}^{\prime \prime} \mid 1 \leq j \leq m\right\}$ is a Steiner tree connecting $S$. Then the trees $T_{i}=P_{i}^{\prime} \cup P_{i}^{\prime \prime}(1 \leq i \leq$ $m)$ are $m$ internally disjoint Steiner trees connecting $S$. 
From Lemmas 2.4, 2.5 and 2.6 we conclude that, for any $S \subseteq V\left(P_{n} \circ H\right)$, there exist $m$ internally disjoint Steiner trees connecting $S$, which implies that $\kappa_{P_{n} \circ H}(S) \geq m$. From the arbitrariness of $S$, we have $\kappa_{3}\left(P_{n} \circ H\right) \geq m=|V(H)|$. The proof of Proposition 2.3 is complete.

Remark 1 . As we have seen, for any $S=\{x, y, z\} \subseteq V\left(P_{n} \circ H\right)$, there exist $m$ internally disjoint Steiner trees connecting $S$ in $P_{n} \circ H$. One can see that only when $x, y, z$ belong to two copies $H\left(u_{i}\right)$ and $H\left(u_{j}\right)$ such that $u_{i} u_{j} \in E\left(P_{n}\right)(1 \leq i \neq j \leq n)$, we use at most one path in $H\left(u_{i}\right)$ or $H\left(u_{j}\right)$. This will be used in Subsection 2.3 to prove our main result Theorem 1.4

\subsection{The Lexicographic product of a tree and a connected graph}

In this subsection, we consider the generalized 3-connectivity of the lexicographic product of a tree and a connected graph, which can be seen as a generalization of the result in the last subsection, and is a preparation of the next subsection.

Proposition 2.7 Let $H$ be a connected graph and $T$ be a tree with $n$ vertices. Then $\kappa_{3}(T \circ H) \geq|V(H)|$. Moreover, the bound is sharp.

Proof: Set $V(T)=\left\{u_{1}, u_{2}, \ldots, u_{n}\right\}$ and $V(H)=\left\{v_{1}, v_{2}, \ldots, v_{m}\right\}$. It suffices to prove that $\kappa_{T \circ H}(S) \geq$ $m$ for any $S=\{x, y, z\} \subseteq V(T \circ H)$, that is, for any $S=\{x, y, z\} \subseteq V(T \circ H)$ there exist $m$ internally disjoint Steiner trees connecting $S$ in $T \circ H$. Recall that $V(T \circ H)=\bigcup_{i=1}^{n} V\left(H\left(u_{i}\right)\right)$. Without loss of generality, let $x \in V\left(H\left(u_{i}\right)\right), y \in V\left(H\left(u_{j}\right)\right)$ and $z \in V\left(H\left(u_{k}\right)\right)$.

If $i, j$ and $k$ are not distinct integers, then there exists a path in $T$ containing $u_{i}, u_{j}$ and $u_{k}$, say $P_{n}$ (We may assume $j=k$. Then $y, z \in V\left(H\left(u_{j}\right)\right)$ ). From Proposition 2.3 and Remark 1, there exist $m$ internally disjoint Steiner trees connecting $S$, which occupies at most one path in $H\left(u_{i}\right)$ or $H\left(u_{j}\right)$ when $x, y, z$ belong to two copies $H\left(u_{i}\right)$ and $H\left(u_{j}\right)$ such that $u_{i} u_{j} \in E\left(P_{n}\right)(1 \leq i \neq j \leq n)$. We now suppose that $i, j$ and $k$ are distinct integers, and that there exists no path containing $u_{i}, u_{j}$ and $u_{k}$. Then there exists a subtree $T^{\prime}$ in $T$ such that $d_{T^{\prime}}\left(u_{i}\right)=d_{T^{\prime}}\left(u_{j}\right)=d_{T^{\prime}}\left(u_{k}\right)=1$ and all the vertices of $T^{\prime} \backslash\left\{u_{i}, u_{j}, u_{k}\right\}$ have degree 2 in $T^{\prime}$ except for one vertex, say $u_{1}$ with $d_{T^{\prime}}\left(u_{1}\right)=3$. Clearly, there is a unique path $P_{1}$ connecting $u_{1}$ and $u_{i}$, a unique path $P_{2}$ connecting $u_{1}$ and $u_{j}$, a unique path $P_{3}$ connecting $u_{1}$ and $u_{k}$. It is clear that $P_{1}, P_{2}, P_{3}$ are three internally disjoint paths. Set $T^{\prime \prime}=T^{\prime} \backslash\left\{u_{i}, u_{j}, u_{k}\right\}$. Obviously, $T^{\prime \prime} \circ H$ is $m$-connected. We have the following four cases to consider.

Case 1. $d_{T^{\prime}}\left(u_{1}, u_{i}\right)=d_{T^{\prime}}\left(u_{1}, u_{j}\right)=d_{T^{\prime}}\left(u_{1}, u_{k}\right)=1$.

Then the trees $T_{r}=x\left(u_{1}, v_{r}\right) \cup y\left(u_{1}, v_{r}\right) \cup z\left(u_{1}, v_{r}\right)(1 \leq r \leq m)$ are $m$ internally disjoint Steiner trees connecting $S$.

Case 2. $d_{T^{\prime}}\left(u_{1}, u_{i}\right) \geq 2$ and $d_{T^{\prime}}\left(u_{1}, u_{j}\right)=d_{T^{\prime}}\left(u_{1}, u_{k}\right)=1$.

Let $u_{i-1}$ be the vertex such that $u_{i-1} u_{i} \in E\left(T^{\prime}\right)$ and $u_{i-1}$ is closer to $u_{1}$ than $u_{i}$ in $P_{1}$. Recall that $T^{\prime \prime} \circ H$ is $m$-connected. From Lemma 2.2 $\left(T^{\prime \prime} \circ H\right) \vee\left\{x, V\left(H\left(u_{i-1}\right)\right)\right\}$ is $m$-connected and hence there exists an $x, U$-fan in $\left(T^{\prime \prime} \circ H\right) \vee\left\{x, V\left(H\left(u_{1}\right)\right)\right\}$, where $U=V\left(H\left(u_{1}\right)\right)$. So there exist $m$ internally disjoint paths $P_{1,1}, P_{1,2}, \cdots, P_{1, m}$ connecting $x$ and $\left(u_{1}, v_{1}\right),\left(u_{1}, v_{2}\right), \cdots,\left(u_{1}, v_{m}\right)$, respectively. Therefore, the trees $T_{j}=P_{1, j} \cup y\left(u_{1}, v_{j}\right) \cup z\left(u_{1}, v_{j}\right)(1 \leq j \leq m)$ are $m$ internally disjoint Steiner trees connecting $S$. Case 3. $d_{T^{\prime}}\left(u_{1}, u_{i}\right) \geq 2, d_{T^{\prime}}\left(u_{1}, u_{j}\right) \geq 2$ and $d_{T^{\prime}}\left(u_{1}, u_{k}\right)=1$.

Let $u_{i-1}$ be the vertex such that $u_{i-1} u_{i} \in E\left(T^{\prime}\right)$ and $u_{i-1}$ is closer to $u_{1}$ than $u_{i}$ in $P_{1}$. Recall that $T^{\prime \prime} \circ H$ is $m$-connected. From Lemma 2.2. $\left(T^{\prime \prime} \circ H\right) \vee\left\{x, V\left(H\left(u_{1}\right)\right)\right\}$ is $m$-connected and hence there exists an $x, U$-fan in $\left(T^{\prime \prime} \circ H\right) \vee\left\{x, V\left(H\left(u_{1}\right)\right)\right\}$. So there exist $m$ internally disjoint paths $P_{1,1}, P_{1,2}, \cdots, P_{1, m}$ connecting $x$ and $\left(u_{1}, v_{1}\right),\left(u_{1}, v_{2}\right), \cdots,\left(u_{1}, v_{m}\right)$, respectively (note that the paths $P_{1,1}, P_{1,2}, \cdots, P_{1, m}$ 
belong to $\left.P_{1} \circ H\right)$. Similarly, there exist $m$ internally disjoint paths $P_{2,1}, P_{2,2}, \cdots, P_{2, m}$ connecting $y$ and $\left(u_{2}, v_{1}\right),\left(u_{2}, v_{2}\right), \cdots,\left(u_{2}, v_{m}\right)$, respectively (note that $P_{2,1}, P_{2,2}, \cdots, P_{2, m}$ belong to $P_{2} \circ H$ ). Therefore, the trees $T_{r}=P_{1, r} \cup P_{2, r} \cup z\left(u_{1}, v_{r}\right)(1 \leq r \leq m)$ are $m$ internally disjoint Steiner trees connecting $S$. Case 4. $d_{T^{\prime}}\left(u_{1}, u_{i}\right) \geq 2, d_{T^{\prime}}\left(u_{1}, u_{j}\right) \geq 2$ and $d_{T^{\prime}}\left(u_{1}, u_{k}\right) \geq 2$.

Similar to the above method, there exist $m$ internally disjoint paths $P_{1,1}, P_{1,2}, \cdots, P_{1, m}$ connecting $x$ and $\left(u_{1}, v_{1}\right),\left(u_{1}, v_{2}\right), \cdots,\left(u_{1}, v_{m}\right), m$ internally disjoint paths $P_{2,1}, P_{2,2}, \cdots, P_{2, m}$ connecting $y$ and $\left(u_{2}, v_{1}\right),\left(u_{2}, v_{2}\right), \cdots,\left(u_{2}, v_{m}\right)$, and $m$ internally disjoint paths $P_{3,1}, P_{3,2}, \cdots, P_{3, m}$ connecting $z$ and $\left(u_{3}, v_{1}\right),\left(u_{3}, v_{2}\right), \cdots,\left(u_{3}, v_{m}\right)$, respectively. Therefore, the trees $T_{r}=P_{1, r} \cup P_{2, r} \cup P_{3, r}(1 \leq r \leq m)$ are $m$ internally disjoint Steiner trees connecting $S$.

From the above arguments, for any $S=\{x, y, z\} \subseteq V(T \circ H)$, there exist $m$ internally disjoint Steiner trees connecting $S$, which implies that $\kappa_{T \circ H}(S) \geq m$. From the arbitrariness of $S$, we have $\kappa_{3}(T \circ H) \geq m=|V(H)|$. The proof is complete.

Remark 2. As we have seen, for any $S=\{x, y, z\} \subseteq V(T \circ H)$, there exist $m$ internally disjoint Steiner trees connecting $S$ in $T \circ H$. One can see that only when $x, y, z$ belong to two copies $H\left(u_{i}\right)$ and $H\left(u_{j}\right)$ such that $u_{i} u_{j} \in E(T)(1 \leq i, j \leq n)$, we use at most one path in $H\left(u_{i}\right)$ or $H\left(u_{j}\right)$. This will also be used in Subsection 2.3 to prove our main result Theorem 1.4

\subsection{The Lexicographic product of two general graphs}

After the above preparations, we are ready to prove Theorem 1.4 in the following subsection.

Proof of Theorem 1.4; Without loss of generality, we set $\kappa_{3}(G)=\ell$. Recall that $V(G)=\left\{u_{1}, u_{2}, \ldots, u_{n}\right\}$, $V(H)=\left\{v_{1}, v_{2}, \ldots, v_{m}\right\}$. From the definition of $\kappa_{3}(G \circ H)$, we need to prove that $\kappa_{G \circ H}(S) \geq \ell m$ for any $S=\{x, y, z\} \subseteq V(G \circ H)$. Furthermore, it suffices to show that there exist $\ell m$ internally disjoint trees connecting $S$ in $G \circ H$. Clearly, $V(G \circ H)=\bigcup_{i=1}^{n} V\left(H\left(u_{i}\right)\right)$. Without loss of generality, let $x \in V\left(H\left(u_{i}\right)\right), y \in V\left(H\left(u_{j}\right)\right)$ and $z \in V\left(H\left(u_{k}\right)\right)$. We have the following three cases to consider.

Case 1. The vertices $x, y, z$ belongs to the same $V\left(H\left(u_{i}\right)\right)(1 \leq i \leq n)$.

Without loss of generality, let $x, y, z \in V\left(H\left(u_{1}\right)\right)$. Since $\delta(G) \geq \kappa_{3}(G) \geq \ell$, it follows that the vertex $u_{1}$ has $\ell$ neighbors in $G$, say $u_{2}, u_{3}, \cdots, u_{\ell+1}$. Then the trees $T_{i, j}=x\left(u_{i}, v_{j}\right) \cup y\left(u_{i}, v_{j}\right) \cup z\left(u_{i}, v_{j}\right)(2 \leq$ $i \leq \ell+1,1 \leq j \leq m)$ are $\ell m$ internally disjoint Steiner trees connecting $S$ in $G \circ H$, as desired.

Case 2. only two vertices of $\{x, y, z\}$ belong to some copy $H\left(u_{i}\right)(1 \leq i \leq n)$.

Without loss of generality, let $x, y \in H\left(u_{1}\right)$ and $z \in H\left(u_{2}\right)$. Since $\kappa(G) \geq \kappa_{3}(G)=\ell$, it follows that there exist $\ell$ internally disjoint paths connecting $u_{1}$ and $u_{2}$ in $G$, say $P_{1}, P_{2}, \cdots, P_{\ell}$. Clearly, there exists at most one of $P_{1}, P_{2}, \cdots, P_{\ell}$, say $P_{1}$, such that $P_{1}=u_{1} u_{2}$. From Remark 1 , there exist $m$ internally disjoint Steiner trees connecting $S$ in $P_{1} \circ H$, which occupies at most one path in $H\left(u_{1}\right)$ or $H\left(u_{2}\right)$. For $P_{i}(2 \leq i \leq \ell)$, there exist $m$ internally disjoint Steiner trees connecting $S$ in $P_{i} \circ H$, which occupies no edge in $H\left(u_{j}\right)(1 \leq j \leq n)$. So the total number of the internally disjoint Steiner trees connecting $S$ is $m+(\ell-1) m=\ell m$, as desired.

Case 3. The vertices $x, y, z$ are contained in distinct $H\left(u_{i}\right) \mathrm{s}$.

Without loss of generality, let $x \in H\left(u_{1}\right), y \in H\left(u_{2}\right)$ and $z \in H\left(u_{3}\right)$. Since $\kappa_{3}(G)=\ell$, it follows that there exist $\ell$ internally disjoint Steiner trees connecting $\left\{u_{1}, u_{2}, u_{3}\right\}$ in $G$, say $T_{1}, T_{2}, \cdots, T_{\ell}$. Observe that $\bigcup_{i=1}^{\ell} T_{i}$ is a subgraph of $G$ and $\left(\bigcup_{i=1}^{\ell} T_{i}\right) \circ H$ is a subgraph of $G \circ H$. If there exist $\ell m$ internally disjoint Steiner trees connecting $S$ in $\left(\bigcup_{i=1}^{\ell} T_{i}\right) \circ H$, then these trees are also $\ell m$ internally disjoint Steiner trees connecting $S$ in $G \circ H$. One can see that $\left(\bigcup_{i=1}^{\ell} T_{i}\right) \circ H=\bigcup_{i=1}^{\ell}\left(T_{i} \circ H\right)$, and for any two tree $T, T^{\prime} \in\left\{T_{i} \mid 1 \leq i \leq \ell\right\}$ we have $(T \circ H) \cap\left(T^{\prime} \circ H\right)=H\left(u_{i}\right) \cup H\left(u_{j}\right) \cup H\left(u_{k}\right)$. From Remark 2 , for 
each tree $T_{i}(1 \leq i \leq \ell)$ there exist $m$ internally disjoint Steiner trees connecting $S$, which occupies no edge in $H\left(u_{j}\right)(1 \leq j \leq n)$. Thus, the total number of the internally disjoint Steiner trees connecting $S$ is $m \ell$, as desired.

From the above arguments, we conclude that, for any $S \subseteq V(G \circ H), \kappa_{G \circ H}(S) \geq \kappa_{\left(\bigcup_{i=1}^{\ell} T_{i}\right) \circ H}(S) \geq$ $m \ell$, which implies that $\kappa_{3}(G \circ H) \geq m \ell=\kappa_{3}(G)|V(G)|$. The proof is complete.

\section{Upper bounds of $\kappa_{3}(G \circ H)$ and $\kappa_{3}(G \square H)$}

Li et al. [29] obtained the following results.

Lemma 3.1 [29] For any connected graph $G, \kappa_{3}(G) \leq \kappa(G)$. Moreover, the upper bound is sharp.

Lemma 3.2 [29] Let $G$ be a connected graph with $n$ vertices. For every two integers $s$ and $r$ with $s \geq 0$ and $r \in\{0,1,2,3\}$, if $\kappa(G)=4 s+r$, then $\kappa_{3}(G) \geq 3 s+\left\lceil\frac{r}{2}\right\rceil$. Moreover, the lower bound is sharp.

From Lemmas $3.1,3.2$ and Theorem 1.2 we can derive a sharp upper bound of the generalized 3connectivity of lexicographic product graphs, which is stated as Theorem 1.5

Proof of Theorem 1.5: From Lemma 3.2, for a connected graph $G$, if $\kappa(G)=4 s+r$, then $\kappa_{3}(G) \geq$ $3 s+\left\lceil\frac{r}{2}\right\rceil$, where $r \in\{0,1,2,3\}$. So $\kappa_{3}(G) \geq 3 \cdot \frac{\kappa(G)-r}{4}+\left\lceil\frac{r}{2}\right\rceil=\frac{3}{4} \kappa(G)-\frac{3}{4} r+\left\lceil\frac{r}{2}\right\rceil$. Therefore, $\kappa(G) \leq$ $\left\lfloor\frac{4}{3} \kappa_{3}(G)+r-\frac{4}{3}\left\lceil\frac{r}{2}\right\rceil\right\rfloor$. From Lemma $3.1, \kappa_{3}(G \circ H) \leq \kappa(G \circ H)$. Furthermore, by Theorem 1.2, we have $\kappa_{3}(G \circ H) \leq \kappa(G \circ H)=\kappa(G)|V(H)| \leq\left\lfloor\frac{4}{3} \kappa_{3}(G)+r-\frac{4}{3}\left\lceil\frac{r}{2}\right\rceil\right\rfloor|V(H)|$, where $r \equiv \kappa(G)(\bmod 4)$. The proof is now complete.

The following example indicates that both the lower bound of Theorem 1.4 and the upper bound of Theorem 1.5 are sharp.

Example 1. Let $G$ is a path of order $n(n \geq 4)$ and $H$ is a path of order 3. Then $|V(G)|=n,|V(H)|=3$ and $\kappa_{3}(G)=\kappa_{3}(H)=1$. Since $\kappa(G)=1$, it follows that $r=1$ and $\kappa_{3}(G \circ H) \leq\left\lfloor\frac{4}{3} \kappa_{3}(G)+r-\right.$ $\left.\frac{4}{3}\left\lceil\frac{r}{2}\right\rceil\right\rfloor|V(H)|=3\left\lfloor\frac{4}{3} \kappa_{3}(G)-\frac{1}{3}\right\rfloor=3$ by Theorem 1.5. From Theorem 1.4, we have $\kappa_{3}(G \circ H) \geq$ $\kappa_{3}(G)|V(H)|=3$. So $\kappa_{3}(G \circ H)=3$. Thus, this is a sharp example for both Theorem 1.4 and Theorem 1.5 .

Let us now turn our attention to the generalized 3-connectivity of Cartesian product graphs. As we know in Theorem 1.3. Li et al. gave a lower bound of $\kappa_{3}(G \square H)$. We now derive an upper bound of $\kappa_{3}(G \square H)$.

From Theorem 1.1, we know that $\kappa(G \square H) \geq \kappa(G)+\kappa(H)$. But we mention that it is incorrectly claimed that $\kappa(G \square H)=\kappa(G)+\kappa(H)$ holds for any connected $G$ and $H$, see [18] (p-308). Let $G$ be a graph obtained from two triangles by identifying one vertex in each of them. Then $\kappa(G)=1$ $\kappa(G \square G)=4>2=\kappa(G)+\kappa(G)$, see [18] (p-309). In [41], S̆pacapan obtained the following result.

Lemma 3.3 [41] Let $G$ and $H$ be two nontrivial graphs. Then

$$
\kappa(G \square H)=\min \{\kappa(G)|V(H)|, \kappa(H)|V(G)|, \delta(G)+\delta(H)\} .
$$

By the above result, we can derive a sharp upper bound of the generalized 3-connectivity of Cartesian product graphs, which is stated as Theorem 1.6 .

Proof of Theorem 1.6: From Lemma 3.2 for a connected graph $G$, if $\kappa(G)=4 s+r_{1}$, then $\kappa_{3}(G) \geq$ $3 s+\left\lceil\frac{r_{1}}{2}\right\rceil$, where $r_{1} \in\{0,1,2,3\}$. So $\kappa_{3}(G) \geq 3 \cdot \frac{\kappa(G)-r_{1}}{4}+\left\lceil\frac{r_{1}}{2}\right\rceil=\frac{3}{4} \kappa(G)-\frac{3}{4} r_{1}+\left\lceil\frac{r_{1}}{2}\right\rceil$, where 
$r_{1} \equiv \kappa(G)(\bmod 4)$. Therefore, $\kappa(G) \leq \frac{4}{3} \kappa_{3}(G)+r_{1}-\frac{4}{3}\left\lceil\frac{r_{1}}{2}\right\rceil$. Similarly, for a connected graph $H$, $\kappa(H) \leq \frac{4}{3} \kappa_{3}(H)+r_{2}-\frac{4}{3}\left\lceil\frac{r_{2}}{2}\right\rceil$, where $r_{2} \equiv \kappa(H)(\bmod 4)$. From Lemma 3.1. $\kappa_{3}(G \square H) \leq \kappa(G \square H)$. Furthermore, by Lemma 3.3, we have $\kappa_{3}(G \square H) \leq \kappa(G \square H)=\min \{\kappa(G)|\nabla(H)|, \kappa(H)|V(G)|, \delta(G)+$ $\delta(H)\} \leq \min \left\{\left\lfloor\frac{4}{3} \kappa_{3}(G)+r_{1}-\frac{4}{3}\left\lceil\frac{r_{1}}{2}\right\rceil\right\rfloor|V(H)|,\left\lfloor\frac{4}{3} \kappa_{3}(H)+r_{2}-\frac{4}{3}\left\lceil\frac{r_{2}}{2}\right\rceil\right\rfloor|V(G)|, \delta(G)+\delta(H)\right\}$. The proof is now complete.

To show the sharpness of the upper bound of Theorem 1.6 we consider the following example.

Example 2. Let $G$ is a path of order $n(n \geq 4)$ and $H$ is a path of order $m(m \geq 4)$. Then $\kappa_{3}(G)=$ $\kappa_{3}(H)=1, \kappa(G)=\kappa(H)=1$ and hence $r_{1}=r_{2}=1$. From Theorem 1.6 $\kappa_{3}(G \square H) \leq \min \left\{\left\lfloor\frac{4}{3} \kappa_{3}(G)+\right.\right.$ $\left.\left.r_{1}-\frac{4}{3}\left\lceil\frac{r_{1}}{2}\right\rceil\right\rfloor|V(H)|,\left\lfloor\frac{4}{3} \kappa_{3}(H)+r_{2}-\frac{4}{3}\left\lceil\frac{r_{2}}{2}\right\rceil\right\rfloor|V(G)|, \delta(G)+\delta(H)\right\}=\min \{n, m, 2\}=2$. It can be checked that for any $S \subseteq V(G \square H)$ and $|S|=3, \kappa(S) \geq 2$, which implies $\kappa_{3}(G \square H) \geq 2$. Thus, $\kappa_{3}(G \square H)=2$ and this is a sharp example for Theorem 1.6

Remark 3. In fact, we can improve the result of Proposition 2.7. From Lemma 3.1 we have $\kappa_{3}(T \circ H) \leq$ $\kappa(T \circ H)=|V(H)|$. Combining this with Proposition 2.7, we have $\kappa_{3}(T \circ H)=|V(H)|$. From Theorem 1.3. one may wonder whether $\kappa_{3}(T \square H)=\kappa_{3}(T)+\kappa_{3}(H)-1$ for a connected graph $H$ and a tree $T$ (note that $\kappa_{3}(T)=\kappa(T)=1$ ). For example, let $T=P_{3}$ and $H=K_{4}$. Then $\kappa_{3}(T)=\kappa(T)=1$ and $\kappa_{3}(H)=2$. One can check that $\kappa_{3}(T \square H)=3>2=\kappa_{3}(T)+\kappa_{3}(H)-1$. So the equality does not hold for the Cartesian product of a tree and a connected graph.

\section{Acknowledgements}

The authors are very grateful to the referees' valuable comments and suggestions, which helped greatly to improve the presentation of this paper.

\section{References}

[1] F. Bao, Y. Igarashi, S.R. Öhring, Reliable broadcasting in product networks, Discrete Appl. Math. 83(1998), 3-20.

[2] L.W. Beineke, R.J. Wilson, Topics in Structural Graph Theory, Cambrige University Press, 2013.

[3] A. Blasiak, R. Kleinberg, E. Lubetzky, Lexicographic products and the power of non-linear network coding, arXiv: 1108.2489 [math.CO] 2013.

[4] J.A. Bondy, U.S.R. Murty, Graph Theory, GTM 244, Springer, 2008.

[5] G. Chartrand, S.F. Kapoor, L. Lesniak, D.R. Lick, Generalized connectivity in graphs, Bull. Bombay Math. Colloq. 2(1984), 1-6.

[6] G. Chartrand, F. Okamoto, P. Zhang, Rainbow trees in graphs and generalized connectivity, Networks 55(4)(2010), 360-367.

[7] X. Cheng, D. Du, Steiner Trees in Industry, Kluwer Academic Publisher, Dordrecht, 2001.

[8] D.P. Day, O.R. Oellermann, H.C. Swart, The $\ell$-connectivity function of trees and complete multipartite graphs, J. Combin. Math. Combin. Comput. 10(1991), 183-192. 
[9] K. Day, A.E. Al-Ayyoub, The cross product of interconnection networks, IEEE Trans. Parallel and Distributed Systems 8(2)(1997), 109-118.

[10] D. Du, X. Hu, Steiner Tree Problems in Computer Communication Networks, World Scientific, 2008.

[11] M. Feng, M. Xu, K. Wang, Identifying codes of lexicographic product of graphs, Electron. J. Combin. 19(4) (2012), 56-63.

[12] P. Fragopoulou, S. G. Akl, Edge-disjoint spanning trees on the star network with applications to fault tolerance, IEEE Trans. Computers 45(2)(1996), 174-185.

[13] M. Grötschel, The Steiner tree packing problem in VLSI design, Math. Program. 78(1997), 265281.

[14] M. Grötschel, A. Martin, R. Weismantel, Packing Steiner trees: A cutting plane algorithm and commputational results, Math. Program. 72(1996), 125-145.

[15] R. Gu, X. Li, Y. Shi, The generalized 3-connectivity of random graphs, Acta Math. Sinica 57(2) (2014), 321-330. (in Chinese).

[16] M. Hager, Pendant tree-connectivity, J. Combin. Theory 38(1985), 179-189.

[17] M. Hager, Path-connectivity in graphs, Discrete Math. 59(1986), 53-59.

[18] R. Hammack, W. Imrich, Sandi Klavžr, Handbook of Product Graphs, Second Edition, CRC Press, 2011.

[19] H. R. Hind, O. R. Oellermann, Menger-type results for three or more vertices, Congressus Numerantium 113(1996), 179-204.

[20] A. Itai, M. Rodeh, The multi-tree approach to reliability in distributed networks, Infor. \& Comput. 79 (1988), 43-59.

[21] S. Ku, B. Wang, T. Hung, Constructing edge- disjoint spanning trees in product networks, Parallel and Distributed Systems, IEEE Trans. Parallel and Disjoited Systems 14(3)(2003), 213-221.

[22] F. Li, Z. Xu, H. Zhao, W. Wang, On the number of spanning trees of the lexicographic product of networks, Sci. China, Ser.F 42(2012), 949-959.

[23] H. Li, X. Li, Y. Mao, On extremal graphs with at most two internally disjoint Steiner trees connecting any three vertices, Bull. Malays. Math. Sci. Soc., in press.

[24] H. Li, X. Li, Y. Mao, Y. Sun, Note on the generalied connectivity, Ars Combin. 114(2014), 193-202.

[25] H. Li, X. Li, Y. Sun, The generalied 3-connectivity of Cartesian product graphs, Discrete Math. Theor. Comput. Sci. 14(1)(2012), 43-54.

[26] $\mathrm{S} . \mathrm{Li}, \mathrm{W} . \mathrm{Li}, \mathrm{X}$. Li, The generalized connectivity of complete bipartite graphs, Ars Combin. 104(2012), 65-79. 
The generalized 3-connectivity of lexicographic product graphs

[27] $\mathrm{S} . \mathrm{Li}, \mathrm{W} . \mathrm{Li}, \mathrm{X} . \mathrm{Li}$, The generalized connectivity of complete equipartition 3-partite graphs, Bull. Malays. Math. Sci. Soc. (2)37(1)(2014), 103-121.

[28] S. Li, X. Li, Note on the hardness of generalized connectivity, J. Combin. Optim. 24(2012), 389-396.

[29] S. Li, X. Li, W. Zhou, Sharp bounds for the generalized connectivity $\kappa_{3}(G)$, Discrete Math. 310(2010), 2147-2163.

[30] X. Li, Y. Mao, On extremal graphs with at most $\ell$ internally disjoint Steiner trees connecting any $n-1$ vertices, accepted by Graphs \& Combin.

[31] X. Li, Y. Mao, Y. Sun, On the generalized (edge-)connectivity of graphs, Australasian J. Combin. 58(2)(2014), 304-319.

[32] C.St.J.A. Nash-Williams, Edge-disjonint spanning trees of finite graphs, J. London Math. Soc. 36(1961), 445-450.

[33] O.R. Oellermann, Connectivity and edge-connectivity in graphs: A survey, Congessus Numerantium 116(1996), 231-252.

[34] O.R. Oellermann, On the $\ell$-connectivity of a graph. Graphs \& Combin. 3(1987), 285-299.

[35] O.R. Oellermann, A note on the $\ell$-connectivity function of a graph, Congessus Numerantium 60 (1987), 181-188.

[36] F. Okamoto, P. Zhang, The tree connectivity of regular complete bipartite graphs, J. Combin. Math. Combin. Comput. 74(2010), 279-293.

[37] K. Ozeki, T. Yamashita, Spanning trees: A survey, Graphs \& Combin. 27(1)(2011), 1-26.

[38] E. Palmer, On the spanning tree packing number of a graph: A survey, Discrete Math. 230(2001), 13-21.

[39] G. Sabidussi, Graphs with given group and given graph theoretical properties, Canadian J. Math. 9(1957), 515-525.

[40] N. A. Sherwani, Algorithms for VLSI Physical Design Automation, 3rd Edition, Kluwer Acad. Pub., London, 1999.

[41] S. S̆pacapan, Connectivity of Cartesian products of graphs, Appl. Math. Lett. 21(2008), 682-685.

[42] D. West, Introduction to Graph Theory (Second Edition), Prentice Hall, 2001.

[43] H. Whitney, Congruent graphs and the connectivity of graphs, Amer. J. Math. 54(1932), 150-168.

[44] C. Yang, J. Xu, Connectivity of lexicographic product and direct product of graphs, Ars Combin. 111(2013), 3-12. 
